Jahrbuch Schweiz - Dritte Welt 1996

\title{
Umfrage zum Thema Sozialklausel bei NRO und Gewerkschaften des Südens
}

Michel Egger and Catherine Schümperli Younossian

\section{(2) OpenEdition}

1 Journals

Electronic version

URL: http://journals.openedition.org/sjep/1355

DOI: $10.4000 /$ sjep. 1355

ISSN: 1663-9677

Publisher

Institut de hautes études internationales et du développement

Printed version

Date of publication: 1 mars 1996

Number of pages: $249-258$

ISSN: 1660-5926

\section{Electronic reference}

Michel Egger und Catherine Schümperli Younossian, « Umfrage zum Thema Sozialklausel bei NRO

und Gewerkschaften des Südens », Schweizerisches Jahrbuch für Entwicklungspolitik [Online], 15| 1996, Online erschienen am: 18 Mai 2013, abgerufen am 08 September 2020. URL : http://

journals.openedition.org/sjep/1355; DOI : https://doi.org/10.4000/sjep.1355 


\title{
Umfrage zum Thema Sozialklausel bei NRO und Gewerkschaften des Südens
}

\author{
Michel Egger, Catherine Schümperli
}

Heute, da die Handelsliberalisierung in vollem Gange ist und die neugeschaffene Welthandelsorganisation (WTO) - welche mehr Deregulierung und verschärften Wettbewerb herbeiführt - ihre Arbeit aufnimmt, wird die folgende Frage besonders aktuell: Soll eine Sozialklausel in die internationalen Handelsbeziehungen eingeführt werden?

Die Frage der Sozialklausel, welche namentlich die Vereinigten Staaten und Frankreich beim Abschluss der Uruguay-Runde im Dezember 1994 aufs Tapet brachten, ist äusserst umstritten. Zwischen den Staaten und Gewerkschaften des Norden einerseits, welche angeblich Sozialdumping bekämpfen wollen, und den Regierungen sowie bestimmten NRO des Südens andererseits, die in dieser Initiative eine Tarnform des Protektionismus befürchten, der sie um ihre komparativen Vorteile bringen könnte, kam es zu heftigen Auseinandersetzungen.

In der Schweiz haben seit Anfang 1995 rund 70 Gewerkschafts-, Entwicklungs-, Umwelt-, Frauen- und Menschenrechtsorganisationen eine „Erklärung“ über die Sozialklausel unterzeichnet, deren Tenor sich an den Standpunkten der grossen Gewerkschaftsbünden auf europäischer und weltweiter Ebene inspiriert. Diese Organisationen betonten damit die Notwendigkeit, Schutzvorkehrungen für den Welthandel vorzusehen; sie betrachten die Sozialklausel als Instrument zur sozialen Regulierung und zum Schutz der grundlegenden Rechte aller Männer, Frauen und Kinder am Arbeitsplatz.

Die Erklärung ist jedoch nur ein erster Schritt. Der Konsens muss nun gefestigt und der Grundsatz in der Realität geprüft werden; konkrete Probleme und Auswirkungen dieser Massnahme auf rechtlicher, politischer und wirtschaftlicher Ebene sind zu untersuchen. In dieser Hinsicht war es insbesondere angesichts der offenkundigen Meinungsunterschiede zwischen Norden und Süden wichtig, unseren Partnern - NRO und Gewerkschaften der nördlichen und südlichen Hemisphäre - den Puls zu fühlen. 
Zu diesem Zweck wurde auf der Grundlage der schweizerischen „Erklärung" ein Fragebogen erstellt und weltweit eine breitangelegte Erhebung durchgeführt. 82 Organisationen antworteten, darunter 67 aus Ländern des Südens und Osteuropas ${ }^{1}$, die Gegenstand dieses Artikels bilden.

\section{Art der befragten Organisationen}

Es schien uns wichtig, genau festzustellen, wer die Organisationen waren, die uns geantwortet haben, ob es sich dabei um Gewerkschaften oder Entwicklungsorganisationen, um kirchliche oder weltliche Organisationen handelt. Diese verschiedenen Kriterien spielen bei der Analyse der Ergebnisse eine Rolle.

Unser Muster umfasst: $48 \%$ nichtkirchliche NRO, 32\% kirchliche NRO, 13\% Gewerkschaften und 7\% Forschungszentren. Die nichtstaatlichen Organisationen machen $80 \%$ der Antworten aus, die Gewerkschaften $13 \%$ und die Forschungszentren $7 \%$. Diese Ergebnisse erklären sich aus der Tatsache, dass die Hilfswerke die Hauptakteure bei der Weitergabe an ihre Partner in den Ländern des Südens waren, während sich die Gewerkschaften weniger aktiv beteiligten. Im Gegensatz zu dem, was die sehr positive Haltung gegenüber der Sozialklausel vermuten liesse, die sich aus unserer Erhebung ergeben hat, machen die kirchlichen Organisationen -bei denen die ethischen Argumente überwiegen- lediglich $32 \%$ unseres Musters aus.

\section{Soll eine Sozialklausel in die internationalen Handelsbeziehungen eingeführt werden?}

Da die Frage in Norden und Süden eine Polemik auslöste und bei bestimmten südlichen NRO auf eine negative Haltung stiess, hatte man von unseren Partnern im Süden entweder sehr differenzierte Meinungen oder insgesamt eher gemischte Gefühle erwartet; dem war aber nicht so. Dass die antwortenden NRO und Gewerkschaften eine Sozialklausel mit überwiegender Mehrheit (91\%) begrüssten (nur 6\% grundsätzliche Ablehnungen, 3\% unentschiedene oder zweideutige Anworten) zeigt einmal mehr, welch tiefe Kluft bisweilen die Regierungen von der Basisbevölkerung trennt.

1. Die Fragebogen gliederten sich wie folgt:

- Afrika: insgesamt 16; Burkina Faso 5, Kamerun 7, Madagaskar 1, Zaire 2, Simbabwe 1;

- Lateinamerika: insgesamt 28; Argentinien 2, Bolivien 2, Brasilien 1, Costa Rica 3, Kolumbien 2, Dominikanische Republik 3, Ecuador 1, Mexiko 1, Nicaragua 8, Panama 1, Paraguay 2, Peru 1, 1 unbekannt;

- Asien: insgesamt 21; Bangladesch 2, Hongkong 1, Indien 4, Philippinen 12, Sri Lanka 2;

- Osteuropa: Rumänien 2.

Aus dem Norden erhielten wir 15 beantwortete Fragebogen (13 aus der Schweiz, 1 aus Frankreich, 1 aus Italien). Der vorliegende Beitrag konzentriert sich auf die Umfrage bei NRO des Südens und Ostens. 
Aus welchen Gründen wurde das Plebiszit durchgeführt?

- Verschiedene Organisationen befürchten, dass die Liberalisierung des Welthandels und der verschärfte Wettbewerb insbesondere in den benachteiligten Sozialsektoren die Arbeitnehmer/innenrechte und bedingungen verschlechtern. Die Sozialklausel - eine wirtschaftspolitische Massnahme - wird als Sicherheitsnetz und als notwendiges Regulierungsmittel gesehen.

- Die Sozialklausel schafft die Voraussetzungen für grössere Gerechtigkeit in den Nord-Süd-Beziehungen; sie beinhaltet ethische Gesichtspunkte, die oft vom Welthandel ausgeklammert werden.

- Die Sozialklausel bildet ein Instrument zur Gewährleistung und zur Förderung der - bereits erworbenen oder noch zu sichernden Menschenrechte am Arbeitsplatz, in der Industrie wie in der Landwirtschaft. In diesem Sinne dürfte sie die Auswirkungen der als „repressiv“ empfundenen Politik des Internationalen Währungsfonds (IWF) mildern.

- Durch die Anerkennung der Übereinkommen und die Förderung der Umsetzung trägt die Sozialklausel zur Stärkung der Internationalen Arbeitsorganisation (IAO) bei.

- Dank der Sozialklausel werden die Gewerkschaften gestärkt und die Tarifabschlüsse mit den Unternehmen und Regierungen verbessert.

- Für eine nachhaltige Entwicklung des Nordens wie des Südens bildet die Sozialklausel eine Conditio sine qua non.

- Die Sozialklausel dient als pädagogische Methode, welche die Sozialpartner (vor allem im Süden) für die wesentlichen Konsequenzen des Welthandels sensibilisieren soll.

Auch unter den Befürwortern der Sozialklausel werden Stimmen laut, die vor der Gefahr eines protektionistischen Einsatzes warnen. Dieses Risiko, das nicht eigentlich mit der Sozialklausel an sich, sondern mit dem jeweiligen Gebrauch zusammenhängt, rechtfertigt jedoch die Ablehnung nicht; vielmehr muss die Umsetzung der Klausel sorgfältig geplant und kontrolliert werden.

Fünf von insgesamt 67 Organisationen lehnen die Sozialklausel rundweg ab. Die von den Gegnern der Sozialklausel vorgebrachten Begründungen sind identisch mit den klassischen Argumenten: drohender Protektionismus; Umsetzung illusorisch, da der politische Wille fehlt; Geltungsbereich auf die Exportindustrie beschränkt; Schwierigkeiten bei der Anpassung der Normen an den jeweiligen Entwicklungsstand der Länder; Schmälerung der nationalen Souveränität, usw.

\section{Kann die Sozialklausel folgende Ziele erreichen?}

- Achtung der Arbeitnehmer/innenrechte,

- Verbesserung der Arbeitsbedingungen, 
- Stärkung der nationalen Gesetzgebung über Arbeit und soziale Sicherheit,

- Bekämpfung sämtlicher Spielarten der Ausbeutung und des Sozialdumpings,

- Appell an das Verantwortungsbewusstsein der multinationalen Konzerne,

- gerechtere Verteilung der Wachstumsgewinne,

- Förderung der Ratifizierung und Umsetzung der IAO-Übereinkommen,

- bessere Information der Verbraucher/innen über die Herstellungsbedingungen von Gütern und Dienstleistungen?

$83,5 \%$ der NRO des Südens und Osteuropas beantworteten diese Frage mit $\mathrm{Ja}, 13,5 \%$ mit Nein, 3\% sind unentschieden. Der im Vergleich zur vorigen Frage höhere Anteil negativer Antworten zeigt, dass einige Organisationen die Sozialklausel zwar im Prinzip befürworten, aber an ihrer tatsächlichen Schlagkraft - hauptsächlich wegen des mangelnden politischen Willens der Regierungen - zweifeln. Andere unterstreichen die Wichtigkeit bestimmter Voraussetzungen, wie z.B. verstärkte Mobilisierung der Basis, Angleichung der Normen an den unterschiedlichen Entwicklungsstand und an die heterogene Arbeitsmarktlage, stärkerer Druck der Regierungen und der NRO des Nordens in den internationalen Gremien und auf bestimmte südliche Regierungen, um die oft ungerechten wirtschaftlichen, politischen und gesellschaftlichen Strukturen zu verändern (s. unten, flankierende Massnahmen).

\section{Soll parallel zur Sozialklausel eine Ökoklausel in den internationalen Handel aufgenommen werden?}

Bekanntermassen herrscht in dieser Frage eine Diskrepanz zwischen den Regierungen des Nordens und jenen des Südens, welche eine Art ÖkoImperialismus befürchten. Angesichts der erheblichen Meinungsunterschiede wäre eigentlich von den südlichen Partnern eine sehr gemässigte oder gar ablehnende Antwort zu erwarten gewesen; erstaunlicherweise traf jedoch das Gegenteil zu: 95,5\% der Befragten votierten für eine Ökoklausel, lediglich $4,5 \%$ stimmten dagegen.

\section{Soll sich die Sozialklausel auf die 7 Basisübereinkommen der IAO abstützen?}

$89,6 \%$ der Organisationen wünschen als Inhalt der Sozialklausel die Mindestnormen der schweizerischen „Erklärung“, die an folgende IAOÜbereinkommen gebunden sind:

- Gewerkschaftsfreiheit und Schutz der Gewerkschaftsrechte (Ü. 87)

- Organisations- und Tarifverhandlungsrecht (Ü. 98)

- Mindestalter für die Zulassung zur Erwerbstätigkeit(Ü. 138)

- Zwangsarbeitsverbot (Ü. 29 und 105)

- Gleiche Entlöhnung für gleichwertige Arbeit (Ü. 100), Diskriminierungsverbot in Beschäftigung und Beruf (Ü. 111). 
Diese sieben Übereinkommen wurden von der höchsten Anzahl Staaten unterzeichnet; sie enthalten die grundlegenden und universellen Rechte des Menschen am Arbeitsplatz. Aus der Perspektive der schweizerischen „Erklärung" wird durch diese Auswahl der Stellenwert der übrigen Übereinkommen über die Arbeit, welche vor allem im Rahmen gewerkschaftlicher Aktionen zu verbessern sind, keineswegs relativiert.

Ungeachtet dieser Präzisierung forderten bestimmte Organisationen die Berücksichtigung zusätzlicher Übereinkommen, wie z.B. Übereinkommen Nr. 158 über Wanderarbeitnehmer (Costa Rica), Nr. 110 über landwirtschaftliche Plantagen (Costa Rica), Nr. 135 über Tarifverhandlungen (Costa Rica, Burkina Faso), Nr. 169 über einheimische Völker und Ethnien (Panama, Bolivien).

\section{Soll die Sozialklausel auf multilateraler, regionaler, bilateraler oder privater Ebene umgesetzt werden?}

In den Antworten der meisten Organisationen wird gewünscht, den „Hebel“ an den verschiedenen Anwendungsebenen „anzusetzen“, um die Arbeiterrechte aufzuwerten, wobei jedoch die multilaterale Ebene besondere Beachtung verdient.

\section{Multilaterale Ebene}

Mit multilateraler Ebene ist die Umsetzung im Rahmen der internationalen Gremien gemeint. $86,6 \%$ unserer befragten Partner sind dafür, $10,4 \%$ dagegen, $3 \%$ sind unentschieden.

Zwei Argumente sprechen für die Einführung einer Sozialklausel auf multilateraler Ebene:

- Mit der Globalisierung der Wirtschaft nimmt das Problem der Achtung der Arbeitnehmerrechte globale Dimensionen an und erfordert daher globale Lösungen.

- Dank der multilateralen Umsetzung kann Druck ausgeübt werden auf die Regierungen zahlreicher Länder, deren innerstaatliche Gesetzgebung noch keine Mindestarbeitsnormen enthält.

Der Widerstand gegen die Einführung einer Sozialklausel auf multilateraler Ebene geht von zwei Schlüsselgedanken aus: von der Vormachtstellung der Industrieländer im internationalen Kräfteverhältnis und von der Schwierigkeit, den regionalspezifischen Anliegen und Realitäten auf multilateraler Ebene Rechnung zu tragen.

$86,6 \%$ der befragten Organisationen äusserten sich für die Einführung einer Sozialklausel auf multilateraler Ebene. Interessant war zu sehen, welche internationale Institution als für die Anwendung der Klausel am geeignetsten betrachtet wurde. Vier Elemente sind unserer Erhebung zu entnehmen: 
1. Keine einzige Organisation möchte die Sozialklausel im Rahmen der WTO einführen. Dieses Ergebnis zeugt von einem tiefen Misstrauen der Regierungen und Gesellschaften des Südens gegenüber der neuen Welthandelsorganisation. Begründet wird die Ablehnung durch die krass dominierende Position der Industrieländer während der Uruguay-Runde des GATT, die materiellen Probleme südlicher Länder, überhaupt an der Diskussion teilzunehmen sowie die Befürchtung, die WTO werde sich vor den Karren der multinationalen Konzerne spannen lassen.

2. $49 \%$ der Organisationen befürworten hingegen die Schaffung eines Bindeglieds zwischen IAO und WTO: zwar verfügen beide Organisationen über Erfahrungen in den fraglichen Bereichen (Arbeitsnormen und Handel), die IAO allein aber ist nicht fähig, der Sozialklausel Geltung zu verschaffen („sie hat sich als machtlos erwiesen“), und die WTO ist wegen der drohenden Beeinträchtigung der Handelsfreiheit nicht daran interessiert. Daraus erklärt sich die Nachfrage nach einer effizienten paritätischen Organisation. Was deren Form angeht, so nennen die Vorschläge unserer Partner drei Möglichkeiten:

- Die IAO könnte die Umsetzung der Sozialklausel überwachen und als Rekursinstanz dienen; die WTO wäre für die Anwendung etwaiger Sanktionen sowie für die Schaffung eines den flankierenden Massnahmen gewidmeten Fonds zuständig. Einige NRO regen an, die Koordinierung der beiden Organisationen durch ein ständiges, mit Vertretern der WTO und der IAO besetztes Sekretariat sicherzustellen; andere befürworten eine internationale Ad-hoc-Kommission, die sich aus Vertretern der Regionen zusammensetzt und Klagen entgegennimmt bzw. weiterleitet. Wie ein Partner aus Panama betont, muss das Kontrollsystem der IAO in jedem Fall (z.B. durch die Bindung ihrer technischen Unterstützung an bestimmte Auflagen) ausgebaut und verstärkt werden.

- Die Schaffung eines ständigen Organs innerhalb der WTO unter der Verantwortung der IAO, das mit der Überprüfung der sozialen Dimension der Handelsliberalisierung betraut ist, stellt eine weitere Variante dar.

- Schliesslich wird die Errichtung eines Forums genannt, wo die beiden Organisationen sich regelmässig zu Diskussionen zusammenfinden mit dem Ziel, in Sachen wirtschaftliche Rentabilität und Arbeitsbedingungen auf doppelter Ebene ein annehmbares Niveau sicherzustellen.

3. Ein Viertel (25\%) der Organisationen wollen die Frage der Sozialklausel allein im Rahmen der IAO behandeln: Für die Internationale Arbeitsorganisation sprechen ihr langjähriges Bestehen, ihr Erfahrungsschatz und ihre dreigliedrige Struktur. Allerdings wird der IAO von den meisten befragten Organisationen mangelnde Effizienz bescheinigt. Daher fordern unsere Partner Reformen, die den IAO-Entscheidungen grössere Rechtsverbindlichkeit verleihen sollen: verstärkte Kontrollen vor Ort, Bindung der technischen Hilfe an bestimmte Auflagen (Konditionalität) usw. 
Der geringe Stimmenanteil zugunsten der IAO veranschaulicht die skeptische Haltung der NRO; bestimmte Nichtregierungsorganisationen prangern gar die "Ineffizienz" der IAO an. Michael Hansenne, Generaldirektor der IAO, bringt in einer Veröffentlichung, ("Des valeurs à défendre, des changements à entreprendre"), die Probleme seiner Organisation an den Tag: „Der springende Punkt ist, ob die IAO die positive Entwicklung des Strebens nach grösseren sozialen Fortschritten auch dann aufrechterhalten kann, wenn die Globalisierung der Wirtschaft und der verschärfte Wettbewerb gegenteilige Konsequenzen herbeiführen“ (S. 58, Ü.). Heute ist die IAO zwischen zwei widersprüchlichen Zielsetzungen hin- und hergerissen: Einerseits möchte sie den freiwilligen Ansatz zum Dialog und zur Zusammenarbeit - ihr Instrument zur Förderung des sozialen Fortschritts, dessen Aufgabe das Vertrauen der Mitglieder zerstören würde - weiterhin pflegen; andererseits will sie die Effizienz ihrer normativen Aktion als regulierende Kraft im Welthandel steigern. Solange die Arbeitsorganisation sich nicht eindeutig entscheidet, welche Rolle sie auf der Bühne der Weltwirtschaft spielen will, werden sich die verschiedenen Akteure kaum uneingeschränkt zu ihr bekennen.

4. $21 \%$ der Organisationen zeigen sich enttäuscht über die bestehenden internationalen Organisationen und befürworten die Schaffung einer neuen Institution, wobei sie sich der grossen Schwierigkeiten eines solchen Vorhabens bewusst sind.

\section{Regionale Ebene}

\section{Stellen Regionalverträge ${ }^{2}$ ein geeignetes Forum zur Einführung der Sozialklausel in Handelsabkommen dar?}

$74,6 \%$ der Organisationen bejahen diese Frage, 3\% verneinen sie, $22,4 \%$ sind unschlüssig. Die Befürworter begründen ihre Unterstützung damit, dass jeder Wirtschaftsblock seine Kriterien im Einklang mit der jeweiligen sozioökonomischen Entwicklung der Region festlegen kann. Eine NRO aus Kamerun betonte, dass "die Bewohner desselben geographischen Raumes ja einer gemeinsamen.Kultur angehören und ähnliche Probleme und Interessen haben“.

2. Die wichtigsten regionalen Wirtschaftsabkommen sind: Europäische Union (EU), Verband Südostasiatischer Staaten (ASEAN), Asiatisch-Pazifische Wirtschaftliche Zusammenarbeit (APEC), Nordamerikanische Freihandelszone (NAFTA), Gemeinsamer Zentralamerikanischer Markt (MCAC), Andengruppe, Gemeinsamer Markt im Süden Lateinamerikas (MERCOSUR), Union économique et monétaire de l'Ouest Africain (UEMOA), Union des Arabischen Maghreb (UMA), Wirtschaftsgemeinschaft Westafrikanischer Staaten (ECOWAS), Union douanière et économique des Etats d'Afrique centrale (UDEAC), Zollunion des Südlichen Afrika (SACU), Entwicklungsgemeinschaft des Südlichen Afrika (SADC), Visegrad-Gruppe, Gemeinschaft Unabhängiger Staaten (GUS). Quelle: Courrier de la planète, Intégration régionale, le jeu des blocs, $n^{0} 28$, mai-juin 1995, 


\section{Bilaterale Ebene}

Lediglich $62,6 \%$ der Organisationen möchten die Sozialklausel in Handelsabkommen zwischen zwei Ländern einführen; $19,4 \%$ sind dagegen, $18 \%$ unentschieden. Die Zurückhaltung erklärt sich aus den ungleichen Wirtschaftsbeziehungen zwischen den Industrie- und Entwicklungsländern und durch das Risiko des Abgleitens in den Protektionismus. Eine indische NRO äusserte sich dazu wie folgt: „Auf bilateraler Ebene droht die Gefahr, dass die Industrieländer ungeachtet und entgegen der objektiven Sachlage auf Diskriminierung, ja gar auf Repressalien gegen Entwicklungsländer zurückgreifen.“

\section{Private Ebene}

Die private Ebene deckt Initiativen ab, welche auf die Verbesserung der Arbeitsbeziehungen abzielen, wie z.B. die Verhaltenskodizes multinationaler Konzerne oder Gütesiegel (Labels)in Sachen „Fair Trade“. Solche Massnahmen lassen sich leichter umsetzen als zwischenstaatliche Entscheidungen und richten sich unmittelbar an die betroffenen Akteure (Unternehmen, Produzenten, Verbraucher).

Bei der Mehrheit der befragten Organisationen (68,6\%) stösst eine privat umgesetzte Sozialklausel auf Zuspruch; 4,5\% sind dagegen, $26,9 \%$ unentschieden. Verhaltenskodizes gelten allgemein als Methode, um das Verantwortungsbewusstsein der Unternehmen wachzurufen und sie dazu zu bewegen, in ihren Investitionsländern - aus denen ihnen ja Gewinne zufliessen - ethische Verhaltensweisen an den Tag zu legen. Trotz der positiven Haltung kommt ein gewisses Misstrauen gegen multinationale Konzerne, welche „häufig zu Druckmitteln greifen, um die Arbeitsgesetzgebung zu verwässern“, zum Ausdruck.

\section{Soll die Sozialklausel sich auf Sanktionen stützen, um die Achtung der grundlegenden Arbeiterrechte durchzusetzen?}

Sanktionen gegen einen souveränen Staat, die ihm den Zugang zu bestimmten Märkten verwehren, stellen derzeit eine völkerrechtliche Ausnahmemassnahme dar; trotzdem halten $90 \%$ der befragten Organisationen die Verhängung von Sanktionen für notwendig, um der Sozialklausel auf multilateraler Ebene Geltung zu verschaffen; $64 \%$ sind einverstanden, die staatliche Entwicklungshilfe bei Verstössen gegen in einem bilateralen Handelsabkommen verankerte Sozialstandards einzuschränken.

"Spitzenreiter" (55\%) unter den verschiedenen Zwangsinstrumenten sind ganz klar gezielte Sanktionen in bezug auf die betroffenen Produkte (Marktzutrittsverbot). Diese sicherlich am besten geeigneten Massnahmen ziehen für ein Land die geringste Diskriminierung nach sich. Vorzug geniesst des weiteren die Methode des Einsatzes der Zollpräferenzen: im positiven Sinne, als Präferenzgewährung (48\%), oder im negativen Sinne, als Präferenzentzug (43\%). Aller- 
dings äussern sich die Organisationen ungleich zurückhaltender zu zwingenderen Massnahmen, die (wie z.B. eine allgemeine Zollerhöhung) gegen ein ganzes Land ergriffen werden.

Allgemein zeigte sich, dass Gewerkschaften und NRO der technischen Unterstützung zugunsten der Entwicklungsländer grossen Wert beimessen. Ferner betonten sie, dass die öffentliche Entwicklungshilfe nicht politisiert werden und nicht diskriminierend wirken dürfe. Dass echte Entwicklungsfortschritte unmöglich sind, solange die Arbeitnehmerrechte mit Füssen getreten werden und nur eine Minderheit von den Anstrengungen der Arbeitenden profitiert, wird von den Organisationen ebenfalls unterstrichen. Unter diesen Voraussetzungen sind $64 \%$ damit einverstanden, die öffentliche Entwicklungshilfe an Auflagen zu knüpfen - vorausgesetzt allerdings, dass nicht die NRO beeinträchtigt werden, sondern nur die zwischenstaatliche Hilfe betroffen ist.

Man mag die Äusserungen einer panamaischen NRO als fatalistisch oder auch als klarsichtig bezeichnen: „Die wirtschaftliche, politische, soziale, kulturelle und technische Entwicklung der armen Länder wird ohnehin durch das Spiel der Weltmächte bestimmt, um nicht zu sagen dirigiert. Uns liegt daran, zu wissen, wer über die Konditionalität der Hilfe entscheidet; gleichzeitig sind wir überzeugt, dass Mindestarbeitsnormen unbedingt beachtet werden müssen. Eine klare Definition der Kriterien und der Verantwortungen sollte verhindern, dass die Konditionalität der Hilfe als wirtschaftliche und politische Waffe missbraucht wird“.

\section{Flankierende Massnahmen}

Schliesslich fragten wir unsere Partner nach den erforderlichen flankierenden Massnahmen, um die Zielsetzung der Sozialklausel in ihren Ländern zu verwirklichen - wobei wir wussten, wie schwierig es ist, eine einheitliche Sozialklausel in einem ungleichen internationalen Umfeld einzuführen.

Drei Massnahmen kristallisierten sich heraus: notwendige Neudefinition der Strukturanpassungsprogramme des Internationalen Währungsfonds, Unterstützung der Basisbewegungen und der Gewerkschaften, Technologietransfer und Entschuldungsmassnahmen. Sehr erstaunlich war, dass die Finanztransfers im Rahmen der öffentlichen Entwicklungshilfe an letzter Stelle genannt wurden.

\section{Welche Rolle sollen die NRO und die Gewerkschaften der südlichen wie der nördlichen Länder bei der Umsetzung der Sozialklausel spielen?}

Auf diese Frage haben unsere Partner aus dem Süden sehr ausführlich geantwortet. Generell sollten die NRO und die Gewerkschaften im Süden wie im Norden wie folgt vorgehen:

- sich aktiv an der Ausarbeitung, Anwendung und Kontrolle der Sozialklausel beteiligen; dazu enger mit der IAO zusammenarbeiten, etwaige Missbräuche und Verletzungen von seiten der Regierungen und Unternehmen verurteilen; 
- Lobbytätigkeit betreiben, um die WTO transparenter und demokratischer zu gestalten;

- gegen Regierungen und Firmen vorgehen, welche sich über die Kriterien der Sozialklausel hinwegsetzen oder sie mit Füssen treten;

- sicherstellen, dass die Interessen der Basis und der Arbeitnehmer/innen gewahrt werden;

- Dialog und Zusammenarbeit zwischen den Entwicklungs-NRO und den Gewerkschaften innerhalb des Landes sowie in den Nord-Süd-Beziehungen ausbauen, z.B. durch die Errichtung von Informationsnetzen, die Lancierung gemeinsamer Kampagnen, Aktionen der gegenseitigen Unterstützung, Definition gemeinsamer Ziele und Stellungnahmen.

\section{Fazit}

Zusammenfassend ${ }^{3}$ stellen wir fest, dass die NRO und die Gewerkschaften des Südens wie Osteuropas, die auf unsere Umfrage reagierten, die Einführung einer Sozialklausel in den Welthandel klar befürworten. Die Sozialklausel stellt für unsere Partner eine wirksame Methode dar, um die Achtung der grundlegenden Arbeitnehmer/innenrechte zu gewährleisten und die Arbeitsbedingungen zu verbessern. Eine oft sehr breite Mehrheit möchte die Sozialklausel, die untrennbar mit einer Ökoklausel verknüpft ist und hauptsächlich auf den sieben Kernübereinkommen der IAO fusst, auf allen Ebenen - multilateral, regional, bilateral, privat - umsetzen; der Schwerpunkt liegt dabei (insbesondere durch die Schaffung einer gemeinsamen IAONWT-Organisation) auf der multilateralen Ebene. Die meisten Organisationen halten Sanktionen wie das Marktzugangsverbot für betroffene Güter und die Konditionalität der Hilfe für notwendig, um der Sozialklausel eine reale Effizienz zu verleihen.

Unsere Ergebnisse - direkte Äusserungen der Basis - heben sich deutlich ab von den Stellungnahmen der südlichen Regierungen, bestimmter grosser NRO des Südens und mancher europäischer Dritte-Welt-Bewegungen.

Die umfassende Erhebung vermittelte uns nicht nur wichtige Erkenntnisse, sondern brachte in den NRO und in den Gewerkschaften des Südens wie des Nordens einen angeregten Überlegungsprozess zum Thema Sozialklausel in Gang.

3. Ein ausführlicher Bericht mit den Stellungnahmen der NRO und der Gewerkschaften der nördlichen Länder, mit Tabellen, Zitaten, dem Erklärungstext und dem Fragebogen kann bei folgender Adresse bestellt werden: Erklärung von Bern, Postfach 212, 1000 Lausanne 9, Tel. 021/624 5417 - Fax 021/624 5419 oder bei: Brot für alle, 3, Chemin du Boisy, 1004 Lausanne, Tel. 021/646 3077 Fax 021/647 6707. 